\title{
Mining epidemiological Dengue Fever Data from Brazil: a gradual pattern based geographical information system
}

\author{
Y. Aryadinata*, Y. Lin**, C. Barcellos***, A.Laurent*, and T.Libourel** \\ *LIRMM, Montpellier, France \\ \{aryadinata, laurent\} @lirmm. fr \\ **UMR ESPACE-DEV (IRD-UM2), Montpellier, France, \\ therese.libourel@univ-montp2.fr, yuan.lin@ird.fr \\ ***Fundaćõ Oswaldo Cruz, Rio de Janeiro, Brazil \\ xrisefiocruz.br
}

\begin{abstract}
Dengue fever is the world's fastest growing vector-borne disease. Studying such data aims at better understanding the behaviour of this disease to prevent the dengue propagation. For instance, it may be the case that the number of cases of dengue fever in cities depends on many factors, such as climate conditions, density, sanitary conditions. Experts are interested in using geographical information systems in order to visualize knowledge on maps. For this purpose, we propose to build maps based on gradual patterns. Such maps provide a solution for visualizing for instance the cities that follow or not gradual patterns.
\end{abstract}

Keywords: Epidemiological Data, Data Mining, Geographic Information Systems, Gradual Patterns.

\section{Introduction}

There are approximately 50 millions new cases each year, and approximately 2.5 billion people live in endemic countries [1] located in the tropical zone between the latitudes of $35^{\circ} \mathrm{N}$ and $35^{\circ}$ [2]. The vector for dengue infection is Aedes aegypti, which has a strictly synanthropic lifestyle [3]. The proliferation of these mosquitoes is supported by both weather patterns and the contemporary style of human life in large cities, where large amounts of water are deposited in the environment and become potential breeding grounds for mosquito reproduction [4]. These factors have been shown to influence the occurrence and spread of dengue infection over the last 50 years.

The incidence of dengue infection has a characteristic seasonal movement in almost all regions of the world, where periods of high transmissibility are experienced during certain months of the year. This phenomenon has been explained by the close relationship between the density variation in winged forms of the vector and climatic conditions [5,6], such as rainfall, temperature and relative humidity.

Since 1986, Brazil has been affected by dengue epidemics that have reached dramatic proportions. This country of continental dimensions has a wide territorial range of 
tropical and subtropical climates (hot and humid), with an average annual temperature above $20^{\circ} \mathrm{C}$ and rainfall exceeding $1,000 \mathrm{~mm}$ per year. These characteristics provide suitable abiotic conditions for the survival of Aedes aegypti [7], the main dengue vector present in the Americas.

From 1990 to 2010, 5.98 million cases of dengue were reported, and autochthonous cases have been recorded in $80 \%$ of the 5,565 Brazilian municipalities. The period of greatest risk for dengue occurrence has been shown to be during or immediately following the rainy season $[5,6]$, and there is a reduced incidence during the remaining months of the year. However, epidemiological studies on the relationship between dengue infection and climate variables in Brazil are scarce.

Studies in wide (national) geographical scales and considering the interactions between spatial and time are still scarce in dengue literature. [3] revealed rapid travelling waves of DHF crossing Thailand emanating from Bangkok every 3 years. Inversely, in Cambodia seasonal propagation waves are originated in poor rural areas being their propagation conditioned by road traffic [8]. In Peru, dengue spatial and temporal dynamics was influenced by the different sociodemographic and environmental among eco-regions [9]. The recent spread of dengue in Brazil is equally related to human mobility across cities network and leaving remote country regions relatively protected [10]. However, unlike contagious diseases, dengue transmission is constrained the environmental substrate on which vector must reproduce and infect people. Thus, the presence and abundance of vector are necessary but not sufficient condition to dengue transmission.

Climate and environmental changes may exacerbate the present distribution of vector borne diseases as well as extend transmission to new niches and populations [11]. Both trends underline the role of health surveillance systems in order to detect and conduct preventive actions in unusual transmission contexts. Climate changes affect populations in different ways and intensities according to the vulnerability of social groups, which is associated to their insertion in place and society. Spatial analysis offers important tools to describe measure and monitor health impact on vulnerable populations under possible scenarios. Brazilian territory presents a wide variety of temperature ranges and rainfall regimes. In addition to climatic variations, unequal urban infrastructure among cities and differential territory occupation patterns increases the complexity of dengue nationwide dynamics.

The important questions arising from experts debate and studies are :

1. Which patterns were more important to explain dengue distribution? What is important? Climate? Sanitary conditions? Human mobility?

2. Which years are typical and regular (the same patterns appear along all years)? And which are abnormal (patterns are different for one atypical year)? For example, extreme climate events, el Niño ?

3. Mapping the patterns. Are patterns concentrated (spatial clusters)? Where are located the different (and contradictory) patterns?

4. Is this spatial pattern related to other geographical features? (relief, ecosystems, roads, rivers, urban regions, etc.).

In this paper, we propose a method using a gradual pattern mining to analyze and to discover the patterns of behavior in dengue fever cases in Brazil. This method also 
allows us to produce a gradual map that can directly be used to see the behavior of the cases of dengue fever from the geographic approach.

In Section 2, we introduce the method to find the gradual patterns, which can be used to create binary and gradual map that described in Section 3. Section 4 presents the data and indicators in terms of analysis and how to produce the binary map (Section 4.2) and the gradual map (Section 4.3). Section 5 concludes the paper and gives the perspectives of our research.

\section{Gradual Patterns}

Gradual pattern mining has been recently introduced as the topic addressing the automatic discovery of gradual patterns from large databases. Such databases are structured over several attributes which domains are totally ordered, considering a relation $\leq$. For instance Table 1 reports an example of such a database.

We consider a toy database containing the information about a disease taken on five cities. Each tuple from the table 1 corresponds to a city, and reports the number of cases for this disease (last column) studied by respect with the number of inhabitants from the city (in thousands), the average humidity (in percentage), and the average income (in $\mathrm{K}$ euros).

Table 1. Database $D$ describing a toy example for a disease in 5 cities

\begin{tabular}{|c|c|c|c|c|}
\hline Id & Nb Inhabitants $(\times 1000)$ & Humidity & Income & Nb Cases $(\times 1000)$ \\
\hline$C_{1}$ & 110 & 53 & 30 & 10 \\
$C_{2}$ & 202 & 71 & 61 & 28 \\
$C_{3}$ & 192 & 64 & 62 & 43 \\
$C_{4}$ & 233 & 83 & 81 & 41 \\
$C_{5}$ & 225 & 75 & 73 & 39 \\
\hline
\end{tabular}

An example of gradual patterns is The higher the number of inhabitants, the higher the degree of Humidity, the higher the number of cases of the disease.

We remind below some concepts of the literature on gradual patterns.

Definition 1. Gradual item. A gradual item is a pair $(i, v)$ where $i$ is an item and $v$ is variation $v \in\{\uparrow, \downarrow\}$. $\uparrow$ stands for an increasing variation while $\downarrow$ stands for $a$ decreasing variation.

Example 1. (NbInhabitants, $\uparrow)$ is an item of graduel item.

Definition 2. Gradual Pattern (also known as Gradual Itemset). A gradual pattern is a set of gradual items, denoted by $G P=\left\{\left(i_{1}, v_{1}\right), \ldots,\left(i_{n}, v_{n}\right)\right\}$. The set of all gradual patterns that can be defined is denoted by GP.

Example 2. $\{($ NbInhabitants, $\uparrow),($ Humidity,$\uparrow),($ NbCases,$\uparrow)\}$ is a gradual itemset. 
Gradual pattern mining aims at extracting the frequent patterns, as in the classical data mining framework for itemsets and association rules.

Definition 3. Given a threshold of a minimum support $\sigma$, a gradual pattern $G P$ is said to be frequent if $\operatorname{supp}(G P) \geq \sigma$.

For describing what frequent means in the context of gradual patterns, several supports have been proposed in the literature. All these materials are based on the idea that it takes the number / proportion of transactions in the database (e.g., the number / proportion of cities in our example) that respect the pattern. For being counted, a transaction must behave adequately with respect to other cities. For example, in Table 2, we see that the number of cases and the number of inhabitants increase together for cities 1 and 2 , as $110<202$ and $10<28$. If the variation is decreasing $(\downarrow)$ then the numbers must follow it. For instance for cities 3 and 4 , the number of inhabitants increases ((Inhabitants, $\uparrow))$ and the number of cases $(($ cases,$\downarrow))$ decreases as $192<233$ and $43>41$.

One of the support proposed in the literature [12] is based on the length of the longest path of cities that can be built using this idea.

Definition 4. The support of gradual pattern GP is given by the following formula : $\operatorname{support}(G P)=\frac{\max _{L \in l}(|L|)}{(|R|)}$.

For determining the longest path, we build the precedence graph for the pattern being considered (Fig. 1). It can be the case that several paths can be built for a pattern, as shown below when trying to order the cities with respect with the pattern $\{($ NbInhabitants,$\uparrow),($ Humidity,$\uparrow),($ NbCases,$\uparrow)\}$. Two orderings are possible: L1 and $L 2$.

Table 2. Two list obtained of $\{($ NbInhabitants, $\uparrow),($ Humidity,$\uparrow),($ NbCases,$\uparrow)\}: L 1$ and $L 2$ (from left to right)

\begin{tabular}{|c|c|c|c|}
\hline Id & Nb Inhabitants & Humidity & Nb Cases \\
\hline$C_{1}$ & 110 & 53 & 10 \\
$C_{2}$ & 202 & 71 & 28 \\
$C_{4}$ & 233 & 83 & 41 \\
\hline
\end{tabular}

\begin{tabular}{|l|c|c|c|}
\hline $\mathrm{Id}$ & Nb Inhabitants & Humidity & Nb Cases \\
\hline$C_{1}$ & 110 & 53 & 10 \\
$C_{2}$ & 202 & 71 & 28 \\
$C_{5}$ & 225 & 75 & 39 \\
\hline
\end{tabular}

Precedence graphs can also be represented in the form of binary matrices as shown in Table 3 for the pattern $\{($ NbInhabitants, $\uparrow),($ Humidity,$\uparrow),($ NbCases,$\uparrow)\} . C_{2}$ precedes $C_{4}$ and $C_{5}$ (value 1 of the matrix), but not $C_{1}$ and $C_{3}$ (value 0 of the matrix). We have here: support $(\{($ NbInhabitants,$\uparrow),($ Humidity,$\uparrow),($ NbCases,$\uparrow)\})=\frac{3}{5}$ as $\mathrm{P}$ is taken by the maximum list of cities $\left\langle C_{1}, C_{2}, C_{4}\right\rangle$ and $\left\langle C_{1}, C_{2}, C_{5}\right\rangle$.

For representing a pattern on the whole database, we consider precedence graphs, as shown by Fig. for the pattern $\{($ NbInhabitants,$\uparrow),($ Humidity,$\uparrow),($ NbCases,$\uparrow$ )$\}$. Such a graph can be represented in a binary matrix, which allows to optimize the computations. For instance, there are two longest paths in this example: $\left\langle C_{1}, C_{2}, C_{4}\right\rangle$ and $<C_{1}, C_{2}, C_{5}>$. The support is thus equal to $\frac{3}{5}$. 


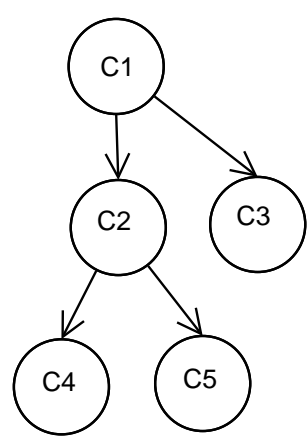

Fig. 1. Precedence graph associated to the pattern $\{($ NbInhabitants, $\uparrow),($ Humidity,$\uparrow$ )$,($ NbCases,$\uparrow)\}$

Table 3. Binary matrix associated to the pattern $\{($ NbInhabitants, $\uparrow),($ Humidity,$\uparrow$ )$,($ NbCases,$\uparrow)\}$

\begin{tabular}{|l|c|c|c|c|c|}
\hline City & $C_{1}$ & $C_{2}$ & $C_{3}$ & $C_{4}$ & $C_{5}$ \\
\hline$C_{1}$ & 1 & 1 & 1 & 1 & 1 \\
$C_{2}$ & 0 & 1 & 0 & 1 & 1 \\
$C_{3}$ & 0 & 0 & 1 & 0 & 0 \\
$C_{4}$ & 0 & 0 & 0 & 1 & 0 \\
$C_{5}$ & 0 & 0 & 0 & 0 & 1 \\
\hline
\end{tabular}

In our work, we aim at displaying such gradual patterns computing from geographical data on maps. For this purpose, we propose to display cities in a form (e.g., color or size) that depends on whether it contributes or not to some gradual pattern. For instance, if the city 1 does not behave the same as all the other cities for pattern (Humidity, $\uparrow),(\mathrm{NbCases}, \uparrow)$ then it will be pointed out be being displayed in red why all the other cities will be colored in green.

\section{Building Binary and Gradual Maps}

In this section, we present novel methods for visualizing gradual patterns on maps. We propose two methods of visualization. Both these methods rely on the calculation of the support with the longest path in the precedence graph

Our idea is to produce maps starting from the extraction of gradual patterns. We will explain this part with a simple example of a region that contains five cities $\left(C_{1}, C_{2}, C_{3}\right.$,$\left.C_{4}, C_{5}\right)$. Based on data from these cities, we believe we have an interesting pattern that we can apply in a map. We then want to know, for each city, how it contributes or not to identify spatial pattern. Then we can represent this information to the user on the maps which he is accustomed. The principle is that each pattern corresponds to a layer in the GIS. Cities are then represented differently depending on their contribution to the cause, i.e., a dichotomous variable. 
Table 4. The list of longest paths associated to Fig. 1

\begin{tabular}{|l|c|}
\hline List & Length \\
\hline$C_{1}, C_{3}$ & 2 \\
$C_{1}, C_{2}, C_{4}$ & 3 \\
$C_{1}, C_{2}, C_{5}$ & 3 \\
\hline
\end{tabular}

We consider two approaches. The first approach, called "binary map" is to represent a color (e.g., green) the cities that contribute to maximum path length on the pattern, and within another color (e.g. red) other cities. The second approach is to represent the intensity of the color cities that are in the longest path and less intense color for cities that are in the shortest path. This is called "gradual map", i.e., a continuous variable.

\subsection{Binary Map}

In this binary map, we identify items through its participation in the gradual pattern (between 0 and 1 ) we use the following steps:

1. Calculating each item of the support in line with the binary matrix

2. Extract lines that have the maximum support

3. Identification (green (1)) cities that are included in the set of itemset length of the maximum path.

4. Identification (red (0)) of the cities that are not in the set of itemset length of the maximum path.

By looking at Figure 1, we know the cities which respect the gradual interesting pattern. For example, Figure 2 shows that the city $C_{5}$ is red, do not fully respect the gradual pattern. In contrast, other cities are green because they are in the longest path

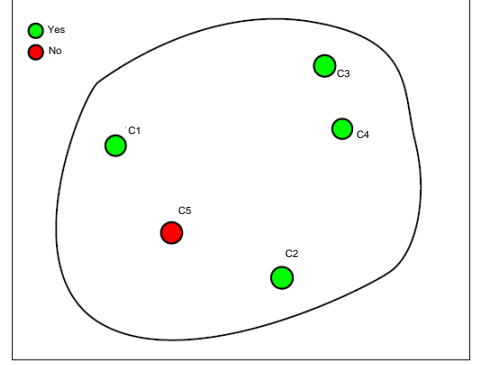

Fig. 2. Binary Map

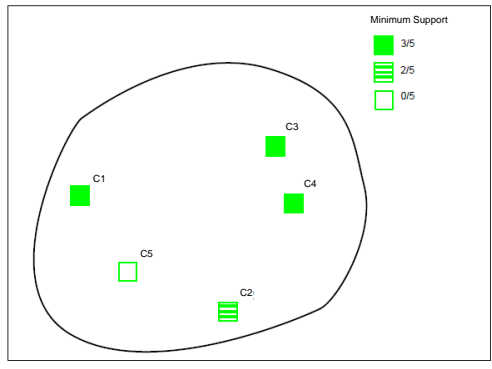

Fig. 3. Gradual Map 


\subsection{Gradual Map}

In order to realize a more detailed maps of the binary map. To do this, we propose a gradual map to visualize the value of the support of each item which corresponds to a proportion of belonging to a support item. To realize this map, we consider the following steps:

1. Calculate the support of each item per line in the binary matrix

2. Take the maximal support size

3. Calculate the intensity of each item in order to identify the importance of which item depends on its intensity value

$$
\text { Intensity }(v)=\frac{\text { The length of support of } v}{\text { The maximum path length }}
$$

4. From the intensity value make the classification using the color indicator on the map.

With this map, we can identify the cities that are more important than others concerning the classification defined in the figure. Thus, the city be more or less illuminated depending on the length of the maximum path in which it appears. For example, in Figure 3, the cities $C_{1}, C_{3}$ and $C_{4}$ are the most illuminated because they belong to the maximum path length.

\section{Experimentation}

Therefore, in our experimentation we use our methods with the dengue cases in Brazil. Firstly, we analyse the data sources and indicators that described in Section 4.1 and Section 4.2 and 4.3 to build the binary and gradual maps.

\subsection{Data sources and Indicators}

Dengue fever (DF) notifications from 2001 to 2012 were summarized by year of symptoms upset and municipality of residence. Data were obtained from the Notifiable Diseases Information System (SINAN acronym in Portuguese), organized by the Brazilian Ministry of Health and freely available in Health Information Department (Datasus). Cases are defined as confirmed Dengue Fever (DF) or Dengue Hemorrhagic Fever (DHF). Cases are confirmed by clinical and laboratory according to standard procedures and submitted to epidemiological investigation by local health surveillance teams. Approximately $30 \%$ of the cases of dengue are also laboratory-confirmed.

The socio-demographic data were obtained from the website of the Brazilian Institute of Geography and Statistics (Instituto Brasileiro de Geografia e Estatstica IBGE; http://mapas.ibge.gov.br). Cities were categorized according to the climate classification map of climate obtained from the Brazilian Institute of Geography and Statistics (IBGE). There are four types of variables on this data: Temperature regime was classified in three categories, Rainfall regime was categorized into four classes, Humidity was categorized into four classes, Sanitary conditions were summarized by the combination of three variables, Mobility was evaluated by means of two variables. 
Individual variables were ranked and the result of summing the ranks was used to categorize into four classes: Very high, High, Medium and Low. All indicators were geocoded and mapped using the coordinates of the city as the center of gravity on the common 5506 existing in 2010. This position was used to create maps and assign information from other layers, such as climate, in a geographic information system (GIS).

During the extraction process of gradual patterns, we cleaned the data so that they do not contain any missing values, false values, etc. In Section 2 , we introduced methods for research on dengue epidemic. Then, we will apply these methods in order to obtain better results.

For simplicity, we choose 25 towns in the state of Rio de Janeiro, Brazil. The State of Rio de Janeiro (RJ) is located east of the southeast region. The capital is Rio de Janeiro. This state has an area of $43909 \mathrm{~km}^{2}$ with about 14,367,000 mainly concentrated along the coast. We choose the state of Rio de Janeiro because of the high frequency of dengue outbreaks in the region. In addition, this region presents a wide morphological diversity (mountains, beaches, dunes, lagoons, etc..). In general, it is divided into three major geographical subregions: the metropolitan lowlands (often called Baixada Fluminense), coastal elevations and northern lowlands. The climate is tropical and the average annual temperature is $23^{\circ} \mathrm{C}$. With this data, we extract the gradual patterns with the method of extraction of conventional gradual patterns on the Section 2.

Table 5 displays some of the interesting gradual patterns in the case of epidemic dengue. After looking at the Table 5 and patterns found, we can infer that the climate (the drought, temperature, humidity) plays the most important role in the case of the dengue epidemic, followed by the level of mobility and sanitation state level.

Table 5. Example of extracted gradual patterns $($ Support $=0.25)$

\begin{tabular}{|l|c|l|}
\hline Motifs & Support & Longest Path \\
\hline$[$ Temp + NbCases +$]$ & 19 & $\begin{array}{l}(330450330550330320330270330555 \\
330510330250330370330430330455 \\
330190330414330490330227330600 \\
330200330220330330)\end{array}$ \\
\hline$[$ Drought + Humid - NbCases -$]$ & 15 & $\begin{array}{l}(330330330200330227330030330490 \\
330190330430330250330510330180 \\
330610330280330320330550330450)\end{array}$ \\
\hline$[$ Drought + Temp - Humid - NbCases -$]$ & 15 & $\begin{array}{l}(330330330200330227330414330190 \\
330430330250)\end{array}$ \\
\hline$[$ Mobility + NbCases -$]$ & 9 & $\begin{array}{l}(330360330220330600330490330455 \\
330250330510330555330270)\end{array}$ \\
\hline$[$ Temp + Mobility - NbCases- $]$ & 7 & $\begin{array}{l}(330330330190330600330227330370 \\
330320330550)\end{array}$ \\
\hline
\end{tabular}

Finally, we can make a binary map and a gradual map that take into account the gradual patterns on the dengue epidemic in Brazil in Fig. 4 and Fig. 5 for the pattern $[$ Temp + Mobility - NbCases- $]$. 


\subsection{Binary Map}

Fig. 4 shows the majority of the cities, following the pattern (Temperature $\uparrow$ )$,($ Mobility,$\downarrow),($ NbCases,$\downarrow)$, displayed in green.

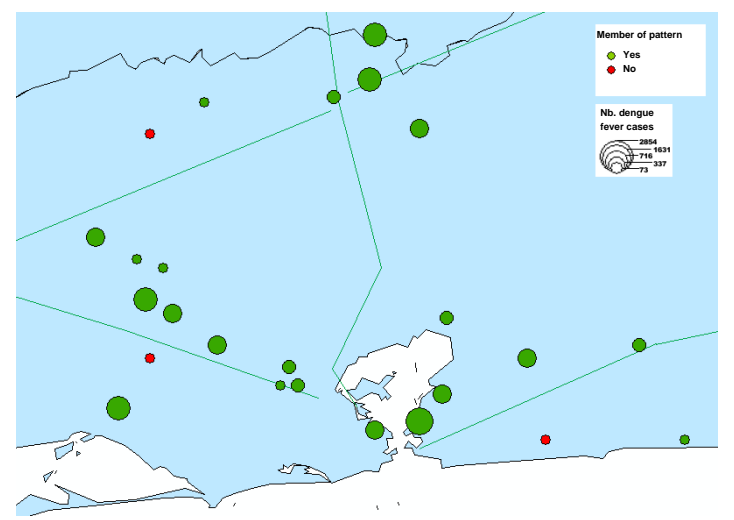

Fig. 4. Binary Map (Temperature,$\uparrow),($ Mobility, $\downarrow),($ NbCases,$\downarrow)$

\subsection{Gradual Map}

In Fig. 5, we present the support level $(0-1)$ of the cities using the intensity color. This map shows more detail information than binary map,Which cities are more related to the pattern (Temperature, $\uparrow),($ Mobility,$\downarrow),($ NbCases,$\downarrow)$. We can see that important emerging epidemic dengue fever mostly appeared in cities located around the coast (shown with the green intensity).

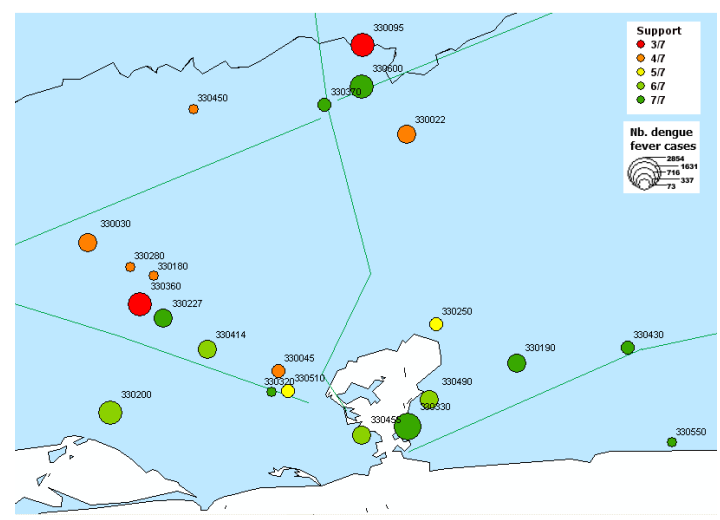

Fig. 5. Gradual Map (Temperature, $\uparrow),($ Mobility,$\downarrow),($ NbCases,$\downarrow)$ 
The difficulty of this step is the production of maps from found gradual patterns, but it is important to retrieve all the items in each pattern corresponding to the length of maximum path.

\section{Conclusion}

In this article, we study how gradual patterns can help to produce maps in geographical information systems. We apply our method in the context of dengue epidemics in Brazil.

Our main perspectives are to merge more criteria for building such maps and to study how a large volume of gradual maps can be displayed to end-users in a userfriendly way by using the so-called layers in geographical information system tools.

\section{References}

1. Organization, W.H.: [The world health report 2006: working together for health]. (2006)

2. Gubler, D., Ooi, E., Vasudevan, S., Farrar, J.: Dengue and Dengue Hemorrhagic Fever. CABI (2013)

3. Gubler, D.J.: Dengue, urbanization and globalization: The unholy trinity of the 21 st century (2011)

4. Kovats, R., Campbell-Lendrum, D., McMichael, A., Woodward, A., Cox, J.: Early effects of climate change: do they include changes in vector-borne disease? Philos Trans R Soc Lond B Biol Sci 356(1411) (2001) 1057-68

5. Souza-Santos, R.: [the factors associated with the occurrence of immature forms of aedes aegypti in ilha do governador, rio de janeiro, brazil]. Rev Soc Bras Med Trop 32(4)

6. Souza-Santos, R., Carvalho, M.: [spatial analysis of aedes aegypti larval distribution in the ilha do governador neighborhood of rio de janeiro, brazil]. Cad Saude Publica 16(1)

7. Yang, H.M., Macoris, M.L., Galvani, K.C., Andrighetti, M.T., Wanderley, D.M.: Dinâmica da transmissao da dengue com dados entomológicos temperatura-dependentes. Tema-Tend. Mat. Apl. Comput 8(1) (2007) 159

8. Teurlai, M., Huy, R., Cazelles, B., Duboz, R., Baehr, C., Vong, S.: Can human movements explain heterogeneous propagation of dengue fever in cambodia? PLoS Negl Trop Dis 6(12) (12 2012) e1957

9. Chowell, G., Cazelles, B., Broutin, H., Munayco, C.V.: The influence of geographic and climate factors on the timing of dengue epidemics in Peru, 1994-2008. Bmc Infectious Diseases 11 (2011) p. 164

10. Catão, R.d.C., Guimarães, R.B.: Mapeamento da reemergência do dengue no brasil-1981/822008. Hygeia 7(13) (2011)

11. McMichael, A., Lindgren, E.: Climate change: present and future risks to health, and necessary responses. J Intern Med 270(5) (2011) 401-13

12. Di-Jorio, L., Laurent, A., Teisseire, M.: Mining frequent gradual itemsets from large databases. In: Proc. of the 8th Int. Symposium on Intelligent Data Analysis 